\title{
ERROR ESTIMATION AND UNAMBIGUOUS RECONSTRUCTION FOR CHINESE FIRST DUAL-CHANNEL SPACEBORNE SAR IMAGING
}

\author{
Tingting Jin ${ }^{\mathrm{a}, \mathrm{b}, *}$, Xiaolan Qiu ${ }^{\mathrm{b}}$, Donghui Hu ${ }^{\mathrm{b}}$, Chibiao Ding ${ }^{\mathrm{b}}$ \\ ${ }^{a}$ University of Chinese Academy of Sciences, Beijing 100049, China - jintingting13@ mails.ucas.ac.cn \\ ${ }^{\mathrm{b}}$ Key Laboratory of Technology in Geospatial Information Processing and Application System, Institute of Electronics, Chinese \\ Academy of Sciences, Beijing 100190, China - (xlqiu, dhhu, cbding)@mail.ie.ac.cn
}

\section{Commission I, WG I/3}

KEY WORDS: Synthetic aperture radar (SAR), high-resolution and wide-swath (HRWS), digital beamforming (DBF), orthogonal subspace method (OSM), unambiguous reconstruction.

\begin{abstract}
:
Multichannel synthetic aperture radar (SAR) is a significant breakthrough to the inherent limitation between high-resolution and wide-swath (HRWS) faced with conventional SAR. Error estimation and unambiguous reconstruction are two crucial techniques for obtaining high-quality imagery. This paper demonstrates the experimental results of the two techniques for Chinese first dualchannel spaceborne SAR imaging. The model of Chinese Gaofen-3 dual-channel mode is established and the mechanism of channel mismatches is first discussed. Particularly, we propose a digital beamforming (DBF) process composed of the subspace-based error estimation algorithm and the reconstruction algorithm before imaging. The results exhibit the effective suppression of azimuth ambiguities with the proposed DBF process, and indicate the feasibility of this technique for future HRWS SAR systems.
\end{abstract}

\section{INTRODUCTION}

Modern remote sensing sets higher requirements on the image resolution as well as the swath width for synthetic aperture radar (SAR) (Currie et al., 1992; Gebert et al., 2009; Krieger et al., 2004; Gebert and Krieger, 2009). Higher azimuth resolution implies higher pulse repetition frequency (PRF), while wider swath requires lower PRF, bringing about an irreconcilable conflict for single-channel SAR. Fortunately, multichannel SAR has been proposed to obtain high-resolution and wide-swath (HRWS) imaging simultaneously, by splitting the antenna into multiple receive channels in azimuth. Low PRF is transmitted to obtain an unambiguous wide swath, then echoes of multiple receivers are combined to improve the azimuth resolution and eliminate azimuth ambiguities.

The feasibility of this mode has been verified by TerraSAR-X and AlOS-2 spaceborne SAR and airborne F-SAR data. J. Kim et al. (2013) demonstrated the first spaceborne experiment of DBF with TerraSAR-X dual receive antenna mode. Gabele et al. (2010) gave the first examples of channel reconstruction for the ground moving target indication (GMTI) capability with TerraSAR-X dual receive channel (DRC) mode. Gebert et al. (2011) and Rosenqvist et al. (2014) conducted airborne experiments of DBF technique with multichannel SAR data. In general, previous study achieved good applications of DBF on multichannel SAR imaging. They applied correlation method (Liu et al., 2013) to estimate the channel mismatches in DBF, with a little deficiency of not considering other estimation algorithms. The orthogonal subspace method (OSM) for error estimation was introduced by Zhang et al. (2010) and Zhang et al. (2015). Yang et al. (2013) and Jin et al. (2016) compared the
OSM algorithm other methods and verified to be effective with simulated and airborne real data. However, there lacks detailed analysis of mechanism of channel imbalances for on-orbit SAR systems. Moreover, there remains a necessary step to complete the spaceborne experiment of error estimation with the OSM.

In this paper, we first discuss various factors of channel mismatches for on-orbit spaceborne SAR systems. We also demonstrate the reconstruction algorithm and subspace-based estimator with Chinese Gaofen-3 DRC mode imaging. Chinese Gaofen-3 DRC mode allows the simultaneous acquisition of echoes by two separated channels of the antenna. Firstly, amplitude and phase errors are estimated from the dual-channel signals. Then, the estimated errors of receive channel 2 are compensated relative to channel 1 . Following is the reconstruction process, which combines two balanced signals into aliasing-free one. Finally, chirp scaling (CS) algorithm is applied to obtain the unambiguous imagery. The primary goal of this paper is to demonstrate the effectiveness of DBF technique on the dual-channel spaceborne SAR mode, providing an approach for future HRWS SAR systems with more channels.

This paper begins with an overview of Gaofen-3 DRC mode in Section 2, with discussion of sources of channel mismatches. In Section 3, the DBF process including error compensation and unambiguous reconstruction is given. The experimental results and some analysis are presented in Section 4. Section 5 draws conclusions and discusses future perspectives.

\footnotetext{
* Corresponding author
} 


\section{GAOFEN-3 DRC MODE AND SIGNAL MODEL}

\subsection{Gaofen-3 DRC Mode}

Gaofen-3 DRC mode, or ultra-fine stripmap mode, is one of the new attempts of Chinese spaceborne SAR systems. This system improves the swath width in stripmap mode without degradation of azimuth resolution.

Figure 1 is a brief illustration of Chinese Gaofen-3 DRC mode. The antenna transmits chirp signals at the center (Tx), and two separate channels $(\mathrm{Rx})$ in azimuth receive echoes simultaneously, shown in Figure 1a. The aperture size and distance between two receive channels are 3.75 meters, shown in Figure 1b. Thanks to dual receive channels, Gaofen-3 ultrafine stripmap mode can achieve approximately $3 \mathrm{~m}$ resolution in azimuth, with PRF less than $2000 \mathrm{~Hz}$. It is difficult to obtain such spatial resolution with the same PRF for the conventional stripmap SAR.

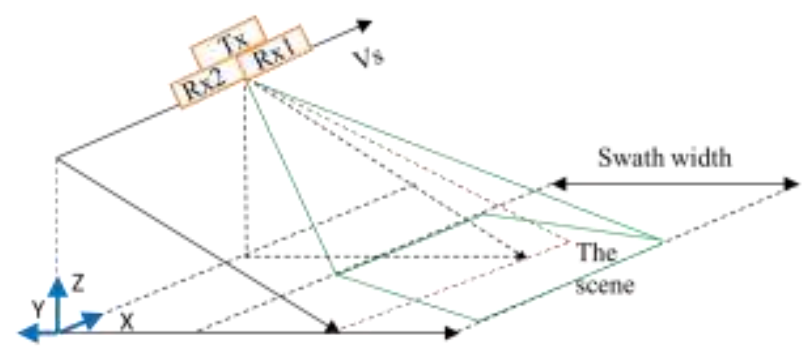

(a)

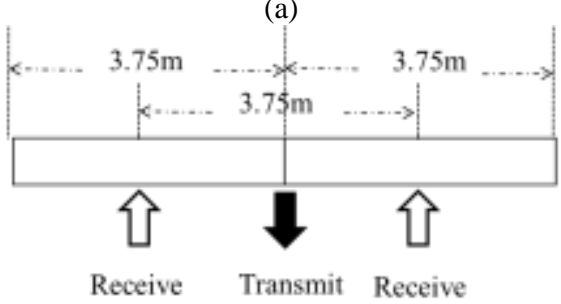

(b)

Figure 1. Illustration of Gaofen-3 DRC mode.

\subsection{Mechanism of Channel imbalances}

Different from single-channel SAR systems, various errors of on-orbit satellite refrain multichannel SAR from high-quality imaging, which makes DBF indispensable. There are three main sources of errors: satellite platform, antenna array and central electronic equipment. Detailed classification of error sources is illustrated in Figure 2. According to the characteristics of errors, they can be divided into three categories, namely: constant errors, random errors and high-frequency jittered errors. Constant errors can be compensated by inner calibration, and high-frequency jittered errors can be neglected because of its harmonic characteristics.

Of all the sources, measure errors of platform velocity and PRF deviation cause non-uniform sampling, making reconstruction indispensable. Other random errors result in magnitude and phase imbalances, which should be estimated and compensated before imaging.

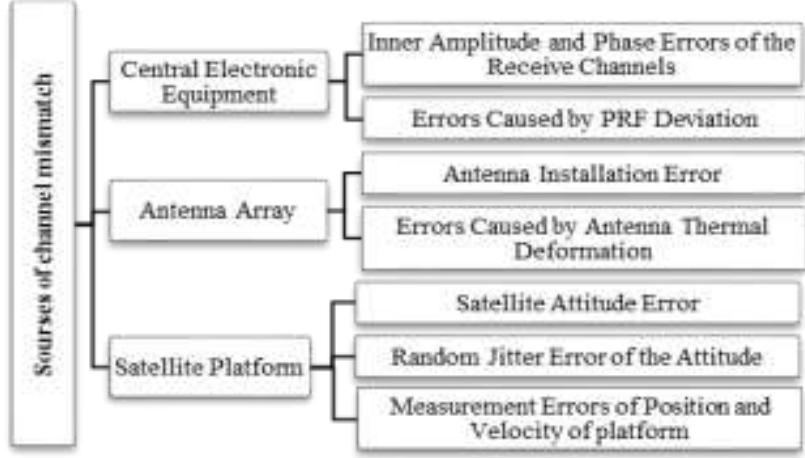

Figure 2. Sources of channel mismatch.

\subsection{Echo Model of DRC Mode}

The geometric model of DRC mode is shown in Figure 1a. The echo received by the $m$-th channel can be expressed as:

$$
\begin{aligned}
S_{m}(\tau, \eta) & =\iint \Gamma_{\mathrm{m}} \exp \left(\mathrm{j} \zeta_{m}\right) \sigma(x, y) h\left(\tau-\frac{2 R_{m}(x, y, z, \eta)}{c}\right) \\
& \cdot g\left(\eta-\frac{x-x_{m}-\Delta x_{m}}{v_{s}}\right) \cdot \exp \left(\frac{-j 4 \pi R_{m}(x, y, z, \eta)}{\lambda}\right) \mathrm{dxdy}
\end{aligned}
$$

where

$$
\begin{array}{ll} 
& R_{m}(x, y, \mathrm{z}, \eta)=\sqrt{\left(x-x_{m}-\Delta x_{m}-v_{s} \eta\right)^{2}+y^{2}+z^{2}} \\
\mathrm{~m} & \text { index of receive channels, } \mathrm{m}=1,2 ; \\
\Gamma_{\mathrm{m}} & \text { total random amplitude error; } \\
\zeta_{m} & \text { total random phase error; } \\
\tau \text { and } \eta & \text { range time and azimuth time, respectively; } \\
\sigma(x, y) & \text { point scattering coefficient at }(x, y, 0) ; \\
h(\tau) & \text { transmitted signal; } \\
g(\eta) & \text { function of antenna pattern, } \\
v_{s} & \text { velocity of the satellite; } \\
x_{m} & \text { azimuth center of the } m \text {-th channel; } \\
\Delta x_{m} & \text { measurement error of azimuth center. }
\end{array}
$$

\section{PROCESSING OVERVIEW}

The processor of Gaofen-3 ultra-fine stripmap mode comprises of two parts: the DBF module and the imaging module, shown in Figure 3. The DBF process contains two techniques: error estimation and compensation, dual-channel signal reconstruction. After DBF processing, chirp scaling (CS) algorithm is applied to produce SAR images with equivalent single-channel signals. We focus on the error estimation and reconstruction algorithm in this section. 


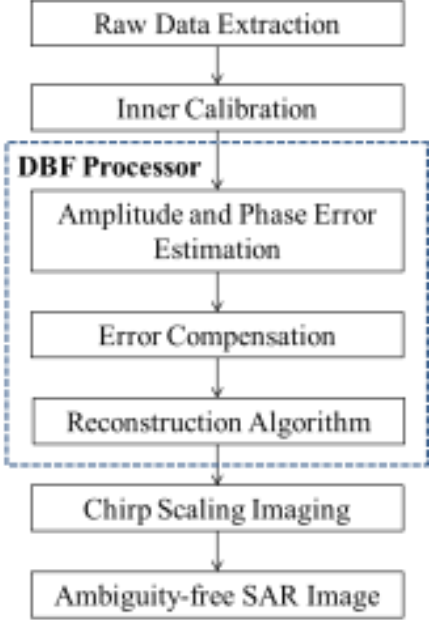

Figure 3. The processor of Gaofen-3 ultra-fine stripmap mode.

\subsection{Estimation of Channel Imbalances}

There possibly exist mismatches in amplitude and phase between two receive channels, causes of which are mentioned above. The amplitude and phase errors will cause degraded resolution and strong ambiguities in the SAR image. The amplitude mismatch between the two channels can be estimated by simple channel balancing, i.e.,

$$
\Gamma_{21}=\sqrt{\frac{E\left\{\left|S_{1}(\tau, \eta) \cdot S_{2}^{*}(\tau, \eta)\right|\right\}}{E\left\{\left|S_{1}(\tau, \eta)\right|^{2}\right\}}},
$$

where $\Gamma_{21}$ is the amplitude offset of channel 2 relative to channel 1 .

The phase mismatch is estimated using the orthogonal subspace method (OSM), which has been evaluated via simulation (Zhang et al., 2010) and applied to airborne multichannel SAR systems (Zhang et al., 2015) without spaceborne application. Jin et al. (2016) has shown that the OSM is an effective estimator for all scenes, with no deterioration for heterogeneous areas. The OSM is based on the orthogonality between the signal subspace and noise subspaces of the sampled covariance matrix. Details of this algorithm are as follows:

Step 1: Conduct the Fourier transform in azimuth of the dualchannel signals in (1). The sampled value is denoted as $X(n)$ :

$$
X(n)=G_{a} A S(n)+u(n), \mathrm{n}=1,2, \ldots, \mathrm{N},
$$

where $\mathrm{N}$ is the number of Doppler bins, $u(n)$ is the additive thermal noise. $A=\left[a_{1}, a_{2}\right]^{T}$ is the array steering vector, $G_{a}$ is a square matrix whose diagonal elements are phase errors in exponential form, i.e.,

$$
G_{a}=\operatorname{diag}\left\{1, \exp \left(j \zeta_{2}\right)\right\}
$$

Step 2: Compute the covariance matrix $R_{X}$ of the sampled signal,

$$
R_{X}(n)=X(n) \cdot X(n)^{H} .
$$

Then conduct eigenvalue decomposition of $R_{X}(n)$ to get the signal subspace $S_{s}$ and the noise subspace $S_{n}$. According to the orthogonal subspace theory, phase errors can be estimated by minimizing the cost function:

$$
J=\underset{G_{a}}{\arg \min }\left(G_{a} \cdot A\right)^{H} S_{n} S_{n}^{H}\left(G_{a} \cdot A\right),
$$

Step 3: Define $w=[1,0]^{T}$, the estimated phase error of channel 2 is:

$$
\zeta_{2}-\zeta_{1}=\left(\widehat{G}_{a}\right)_{22}
$$

where

$$
\widehat{G}_{a}=\operatorname{diag}\left(\frac{\Omega_{1}^{-1} w}{w^{T} \Omega_{1}^{-1} w}\right),
$$

and

$$
\Omega_{1}=(\operatorname{diag}(A))^{H} S_{n} S_{n}^{H}(\operatorname{diag}(A)) .
$$

Finally, average the estimated phase errors by N Doppler bins to improve accuracy.

This algorithm can estimate phase errors from the echoes of static scenes without additional calibration sources. Besides, it only needs inversion of small scale matrixes, thus is efficient. In addition, the OSM can estimate phase errors that vary with azimuth time.

\subsection{Reconstruction Algorithm}

According to the nonuniform sampling theory (Krieger et al., 2004), the optimal PRF entailing uniform sampling meets,

$$
P R F_{u n i}=\frac{2 \cdot v_{s}}{M \cdot D_{a}}
$$

where $M$ is the number of channels, $D_{a}$ is the aperture size of a single channel. However, due to the inconstancy of satellite velocity and the diversity of $P R F$, the practical DRC system can hardly satisfy Equation (11).

The reconstruction algorithm designs a reasonable pre-filter according to the signal model and restores the normal stripmap SAR data. The reconstruction filter is the inverse of the prefilter. The reconstruction filter compensates for the phase shift caused by spatial sampling and antenna displacement, thus applies to non-uniformly sampled data.

For the dual-channel system, the pre-filter matrix for the additional shift is:

$$
\mathbf{H}\left(f_{d}\right)=\left[\begin{array}{ll}
H_{11}\left(f_{d}\right) & H_{12}\left(f_{d}+f_{P R F}\right) \\
H_{21}\left(f_{d}\right) & H_{22}\left(f_{d}+f_{P R F}\right)
\end{array}\right],
$$

where

$$
H_{i j}\left(f_{d}\right)=\exp \left\{j \cdot \frac{\pi}{2 v_{s}} \cdot D_{a} \cdot\left(f_{d}+(j-1)\right) \cdot f_{P R F} \cdot(-1)^{i+1}\right\},
$$

for $i, j=1,2, f_{d}$ is the Doppler frequency, and $f_{P R F}$ denotes the 
PRF. The reconstruction filter $\mathbf{P}$ is obtained from the inverse of the pre-filter matrix, i.e.,

$$
\mathbf{P}=\mathbf{H}^{-1}
$$

After derivation, the $i j$-th element of $\mathbf{P}$ is expressed as:

$$
P_{i j}\left(f_{d}\right)=\frac{(-1)^{i-j} \cdot \exp \left\{j \frac{\pi}{2 v_{s}} D_{a} \cdot\left(f_{d}+2^{i-1}-2(\mathrm{i}-1)\right) \cdot f_{P R F} \cdot(-1)^{j}\right\}}{2 \sin \left(\frac{\pi}{2 v_{s}} \cdot D_{a} \cdot f_{P R F}\right)}
$$

Finally, the equivalent single-channel stripmap data is given by:

$$
S\left(f_{d}\right)=\sum_{i=1}^{M} S_{i}\left(f_{d}\right) \cdot P_{i}\left(f_{d}\right)
$$

The DBF module is completed by error compensation and reconstruction process, so that the band-limited signals are concatenated in the Doppler domain without aliasing.

\section{EXPERIMENT WITH GAOFEN-3 DRC MODE}

\subsection{Results of Error Estimation and Dual-channel Imaging}

The parameters of the DRC mode are listed in Table 1. According to the satellite velocity and the aperture size, the ideal PRF satisfying uniform sampling is $2018.53 \mathrm{~Hz}$. The practical $1877.1 \mathrm{~Hz}$ PRF leads to a separation of $2.015 \mathrm{~m}$ between two equivalent phase centers. The corresponding spatial sampling is illustrated in Figure 4, compared with the ideal uniform sampling. Figure $4 \mathrm{a}$ corresponds to the uniform sampling, where distance between two successive spatial samples is $1.875 \mathrm{~m}$. In reality, the signals are non-uniformly sampled, as shown in Figure 4b.

Here, we present the imaging result of DBF processing, which is elaborated in Section 3. To verify the performance of the OSM, the estimated mismatches between two channels for four typical scenes are listed in Table 2. Scene 1 is the border region between the land and large scale sea, scene 2 is an urban area including strong scattering points, scene 3 is the sea surface, and scene 4 is a mountainous area. As the ambiguities of scene 1 and scene 2 are more obviously visible, we demonstrate the imaging results before and after DBF for the two areas in Figure 5 and Figure 6, respectively. Scene 1 is the border between Qidong City of Jiangsu Province and the Yellow Sea.

The overall scattering of sea surface is weak, and the scattering of mountainous area is homogeneous, the effect of DBF for scene 3 and scene 4 are not visually apparent. So the imaging results of the two scenes are not presented here.

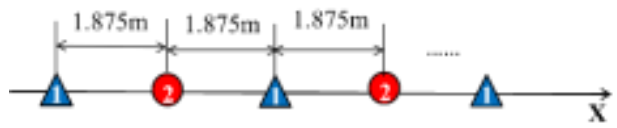

(a)

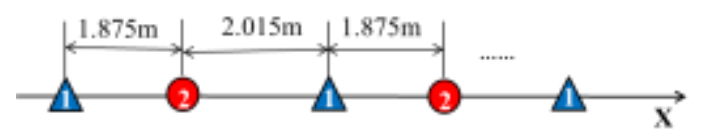

(b)

Figure 4. Illustration of spatial sampling: (a) uniform sampling, and (b) practical sampling
Table 1. Imaging Parameters.

\begin{tabular}{c|cc}
\hline Parameter & Symbol & Value \\
\hline Wavelength & $\lambda$ & $0.05556 \mathrm{~m}$ \\
Look Angle & $\theta$ & 28.81 degrees \\
PRF & $f_{P R F}$ & $1877.7 \mathrm{~Hz}$ \\
Doppler Bandwidth & $B_{d}$ & $2470.53 \mathrm{~Hz}$ \\
Satellite Velocity & $v_{s}$ & $7569.5 \mathrm{~m} / \mathrm{s}$ \\
Sample Frequency & $f_{s}$ & $133.33 \mathrm{MHz}$ \\
Bandwidth & $B_{r}$ & $80 \mathrm{MHz}$ \\
Pulsewidth & $T_{r}$ & $54.99 \mathrm{us}$ \\
\hline
\end{tabular}

Table 2. Estimated channel mismatches

\begin{tabular}{c|cc}
\hline Scenes & $\begin{array}{c}\text { Estimated Amplitude } \\
\text { Error }\end{array}$ & $\begin{array}{c}\text { Estimated Phase Error } \\
\text { (degree) }\end{array}$ \\
\hline Scene 1 & 1.1415 & 14.540 \\
Scene 2 & 1.1250 & 14.657 \\
Scene 3 & 1.1777 & 14.494 \\
Scene 4 & 1.1661 & 15.249 \\
\hline
\end{tabular}

\subsection{Analysis}

From the amplitude and phase offsets in Table 2, the channel mismatches estimated by different scenes are very close, which verifies the system stability of the Gaofen-3 satellite.

In Figure 5a, the azimuth ambiguities of the land are rather obvious due to the weak sea clutter. On the contrary, the ambiguities are much suppressed in Figure 5b. In Figure 6a, the ambiguities of strong scattering points seriously affect the imaging quality of urban area, and the quality is much improved after DBF processing in Figure $6 \mathrm{~b}$.

To quantitatively describe the effect of the DBF technique on the imaging results, we compute the azimuth ambiguity-tosignal ratio (AASR) of the strong scattering points. AASR means the ratio of the power of ambiguous target to the power of real target in the imagery, i.e.,

$$
\operatorname{AASR}=10 \cdot \log \frac{P_{\mathrm{A}}}{S},
$$

where $P_{\mathrm{A}}$ is the power of the ambiguous target, and $\mathrm{S}$ is the power of the real one.

Figure 7a,b correspond to the selected areas in Figure $5 \mathrm{a}, \mathrm{b}$, respectively. As can be seen in Figure 7a, there are five strong scattering points in the selected area. The false targets are evident in Figure 7a along the azimuth below the real ones, and are suppressed in Figure $7 \mathrm{~b}$ after DBF processing. We compute the AASRs of the five targets before and after DBF, respectively. Then average the AASRs of the five targets to get a mean value. Finally, the AASR is $-15.3 \mathrm{~dB}$ before DBF, and $35.6 \mathrm{~dB}$ after DBF processing. Thus the OSM estimator combined with the reconstruction filter can achieve a remarkable improvement in the imaging quality. 

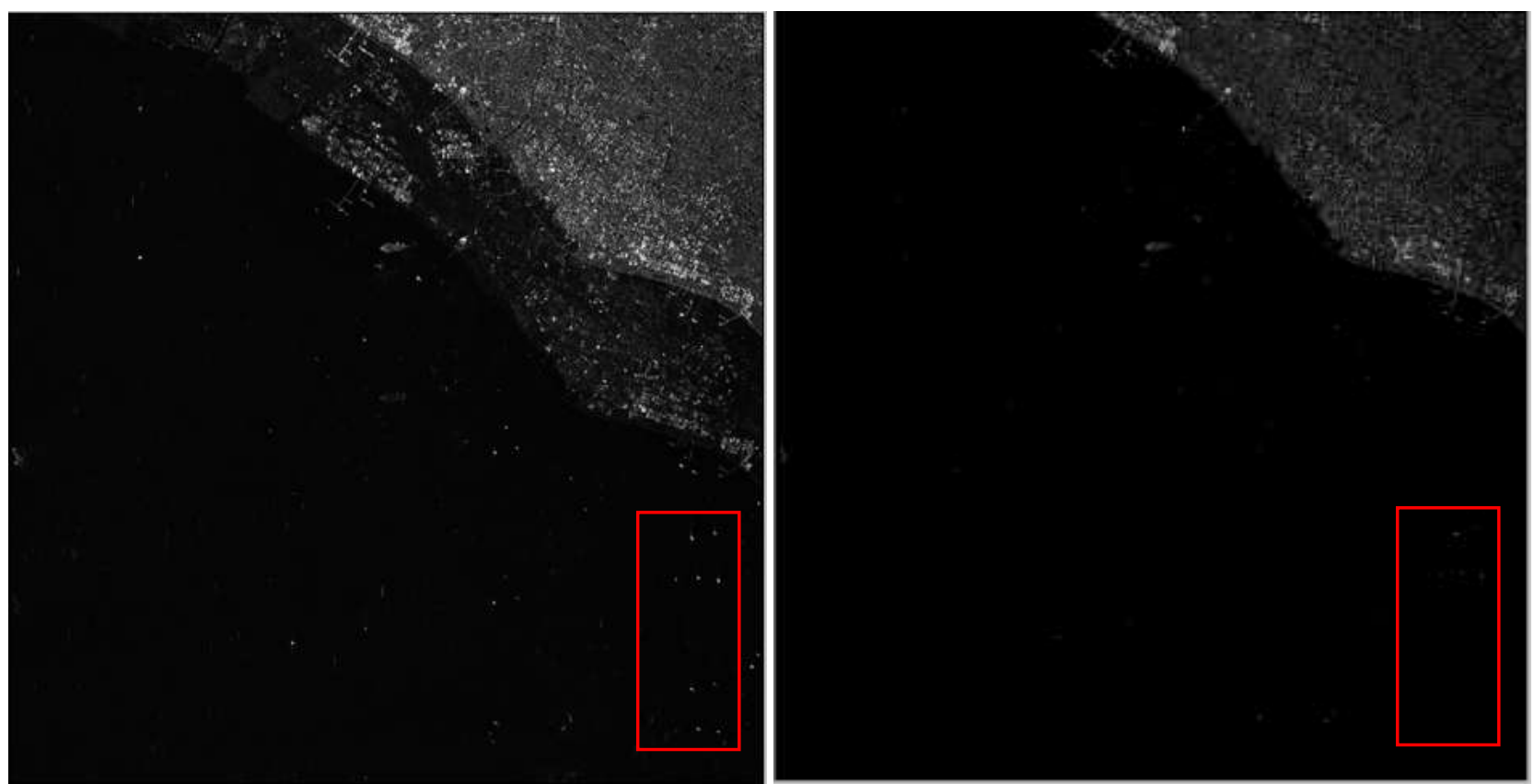

(a)

(b)

Figure 5. Imaging results of Scene 1: (a) before DBF processing, and (b) after DBF processing.

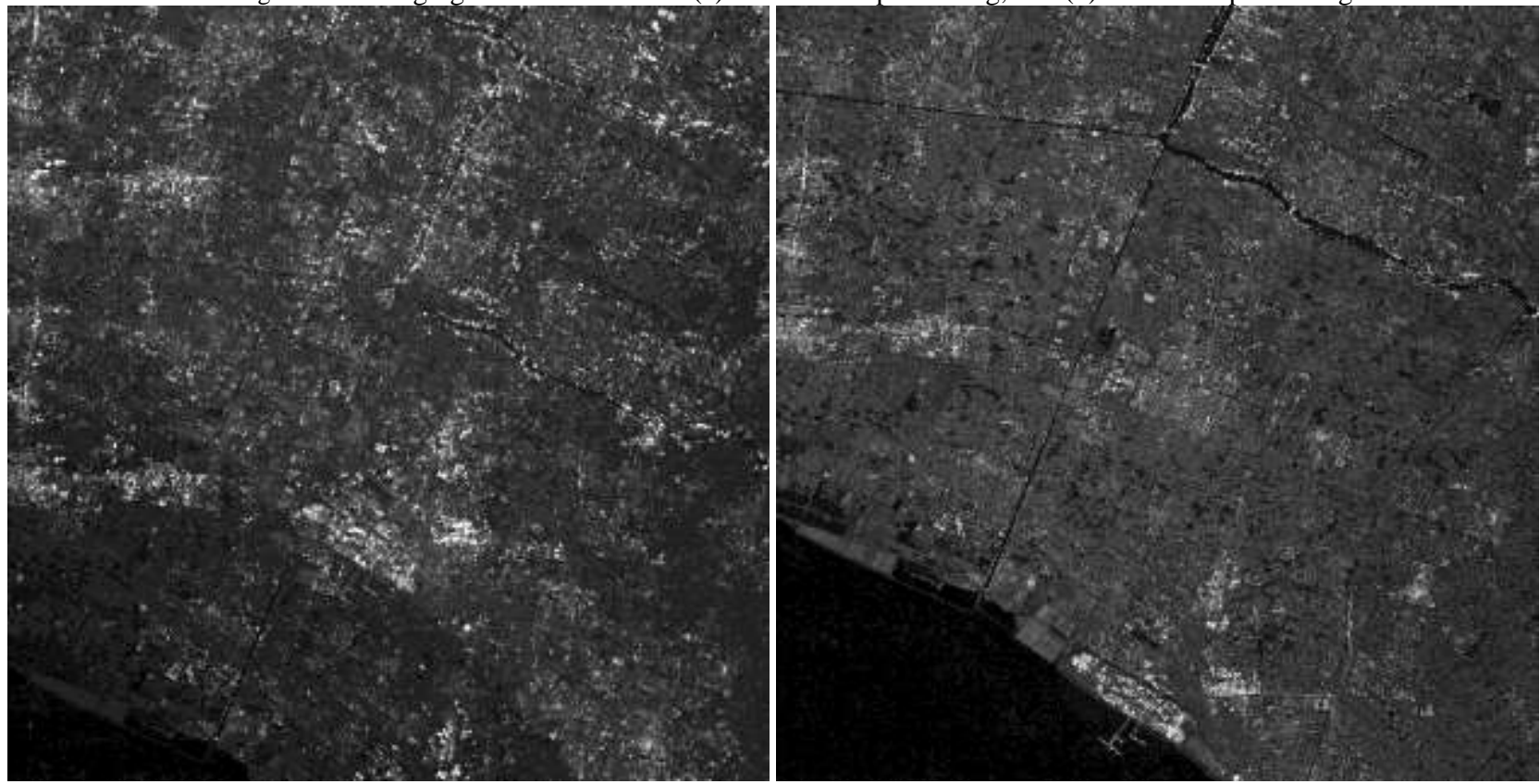

(a)

(b)

Figure 6. Imaging results of Scene 2: (a) before DBF processing, and (b) after DBF processing. 


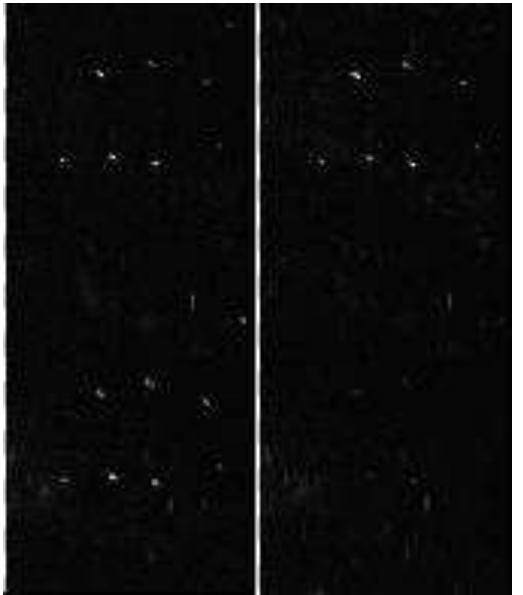

Figure 7. Enlargements of the selected areas in Figure 5: (a) corresponding to Figure 5a, and (b) corresponding to Figure 5b.

\section{CONCLUSION}

The DBF technique with error estimation and reconstruction is successfully applied to Chinese first dual-channel spaceborne SAR imaging. This paper describes the mechanism of channel imbalances for on-orbit SAR systems. More importantly, the orthogonal subspace method is first applied to spaceborne multichannel SAR to estimate phase errors. After error compensation and reconstruction, the results demonstrate a good suppression of azimuth ambiguities. The proposed DBF technique has great potential for application in future HRWS SAR systems.

\section{ACKNOWLEDGEMENTS}

This work was supported by the National Science Foundation of China under Grant 61331017, and the Fundamental Research Project under Grant 41-Y20A13-9001-15/16.

\section{REFERENCES}

Currie, A., and Brown, M.A., 1992. Wide-swath SAR. Proc. Inst. Elect. Eng. F-Radar Signal Process., 139(2), pp. 122135 .

Gebert, N., Krieger, G., and Moreira, A., 2009. Digital beamforming on receive: Techniques and optimization strategies for high-resolution wide swath SAR imaging. IEEE Trans. Aerosp. Electron. Syst., 45(2), pp. 564-592.

Gebert, N., and Krieger, G., 2009. Azimuth phase center adaptation on transmit for high-resolution wide-swath SAR imaging. IEEE Geosci. Remote Sens. Lett., 6(4), pp. 782-786.

Gabele, M., Bräutigam, B., Schulze, D., Steinbrecher, U., TousRamon, N., and Younis, M., 2010. Fore and aft channel reconstruction in the TerraSAR-X dual receive antenna mode. IEEE Trans. Geosci. Remote Sens., 48(2), pp. 795-806.

Gebert, N., Almeida, F., and Krieger, G., 2011. Airborne demonstration of multichannel SAR imaging. IEEE Geosci. Remote Sens. Lett., 8(5), pp. 963-967.
Jin, T.T., Qiu, X.L., Hu, D.H., and Ding, C.B., 2016. Estimation accuracy and Cramer-Rao lower bounds for errors in multichannel HRWS SAR systems. IEEE Geosci. Remote Sens. Lett., 13(12), pp. 1772-1776.

Krieger, G., Gebert, N., and Moreira, A., 2004. Unambiguous SAR signal reconstruction from non-uniform displaced phase center sampling. IEEE Geosci. Remote Sens. Lett., 1(4), pp. 260-264.

Kim, J., Younis, M., Prats, P., Gabele, M., and Krieger, G., 2013. First spaceborne demonstration of digital beamforming for azimuth ambiguity suppression. IEEE Trans. Geosci. Remote Sens., 51(1), pp. 579-590.

Liu, Y.Y. Li, Z., Suo, Z., and Bao, Z., 2013. A Novel Channel Phase Bias Estimation Method for Spaceborne Along-track Multi-channel HRWS SAR in Time-domain. In IET Radar. Conf, Adelaide, Australia, Sep. 2013, pp. 1-4.

Rosenqvist, A.; Shimada, M.; Suzuki, S.; et al., 2014. Operational performance of the ALOS global systematic acquisition strategy and observation plans for ALOS-2 PALSAR-2. Remote Sens. of Environ., 155, pp. 3-12.

Yang, T.L., Li, Z.F., Liu, Y.Y., Suo, Z., and Bao. Z., 2013. Channel error estimation methods for multichannel SAR systems in azimuth. IEEE Geosci. Remote Sens. Lett., 10(3), pp. 548-552.

Zhang, L., Xing, M.D., Qiu, C.W., and Bao. Z., 2010. Adaptive two-step calibration for high-resolution and wide-swath SAR imaging. IET Radar Sonar Navig., 4(4), pp. 548-559.

Zhang, D.D., Qiu, X.L., Hu, D.H., and Ding, C.B., 2015. A subspace-based channel calibration algorithm for geosynchronous satellite-airborne bistatic multi-channel radars. IET Radar Sonar Navi., 8(9), pp. 1008-1017. 